\title{
Cartesian Pre-distortion using a Sigma Delta Modulator for Multi-Standard RF Power Amplifiers
}

\author{
Keith Finnerty, John Dooley and Ronan Farrell
}

Callan Institute, Department of Electronic Engineering, National University of Ireland Maynooth.

email :kfinnerty@eeng.nuim.ie,jdooley@eeng.nuim.ie,rfarrell@eeng.nuim.ie

\begin{abstract}
The system implements a pre-distortion algorithm for a non-linear PA by placing a pseudo static forward model of a PA in the feedback path of a Sigma Delta Modulator. Performing the predistortion in this way reduces the computation overhead incurred in calculating pre-distorter weights for a given power amplifier. In this paper a Cartesian LUT is used to represent the PA model, allowing the system to compensate for PA non-linearity caused by multiple signal standards. The system was tested using 3 modulation standards and showed improvement of up to $28 \mathrm{~dB}$ while the PA model remained constant.
\end{abstract}

Keywords - Sigma Delta, Predistortion, Power amplifiers, Multi-standard.

\section{INTRODUCTION}

With the increasing demand for higher data rates in wireless communications, the modern transceiver must implement spectrally efficient modulation schemes over large bandwidths. A key component of the transceiver system is the Power Amplifier (PA) which increases the power of signals before transmission. In order to improve spectral efficiency the channels in modern communication schemes have reduced guard bands, therefore to prevent interference the PA must be highly linear to avoid spectral spreading. To maintain linearity especially over large bandwidths the input to the PA often must be reduced to ensure it remains linear, this can greatly impact on the efficiency of the amplifier. Predistortion techniques can be used to reduce the non-linear effects allowing spectral efficiency to be achieved and in some cases the range of operation of the amplifier is also increased.

Transceiver systems are currently operating with DSP's and FPGA hardware which allows the transceiver to be adaptable, moving the digital boundary closer to the PA. Advances in digital hardware and the inclusion of this hardware in the transceiver chain means that Digital pre-distortion (DPD) is being used more and more in modern communication systems. Implementation of DPD on FPGA's enables the dynamic reconfiguration of the pre distortion algorithm to deal with the change in PA characteristics when switching between multiple signal standards. Current DPD methods such as AMAM AM-PM and Volterra series require calculations to be performed on the captured data to determine the coefficients for pre distortion, this can be a computationally intensive process which can increase system complexity and impact negatively on the efficiency of the overall system.

The pre distortion method presented in [1] uses a dynamically reconfigurable pre distortion method based on a Cartesian look up table (LUT). There is a feedback path from the output of the PA to the LUT. The weights can then be updated by implementing a training algorithm in the structure however this requires the system to be taken offline for a brief period of time and the calculations can be computationally intensive.

Presented in $[2,3]$ is a method of implementing pre distortion using a sigma delta modulator(SDM), the structure can be seen in Figure 1. The system in Figure 1 is equivalent to the Class-T audio amplifiers developed by Tripath [4]. In the Tripath system a sigma delta is used to drive a class D amplifier for audio applications. The distortion for the amplifier is reduced by using the output of the $\mathrm{PA}$ as the feedback path of the modulator. For RF systems the feedback path would introduce a substantial delay. Modifying the path feedback by using a model for the PA instead of using an output directly from the PA, not only reduces system delay but also the need for high speed ADC's in the feedback path of the system. The benefit of this system is that it removes the need to pre calculate the pre distortion coefficients requiring only the forward model of the PA.

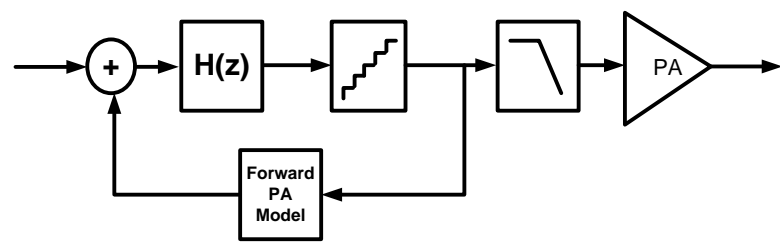

Figure 1 Digital pre-distortion using sigma delta modulators 


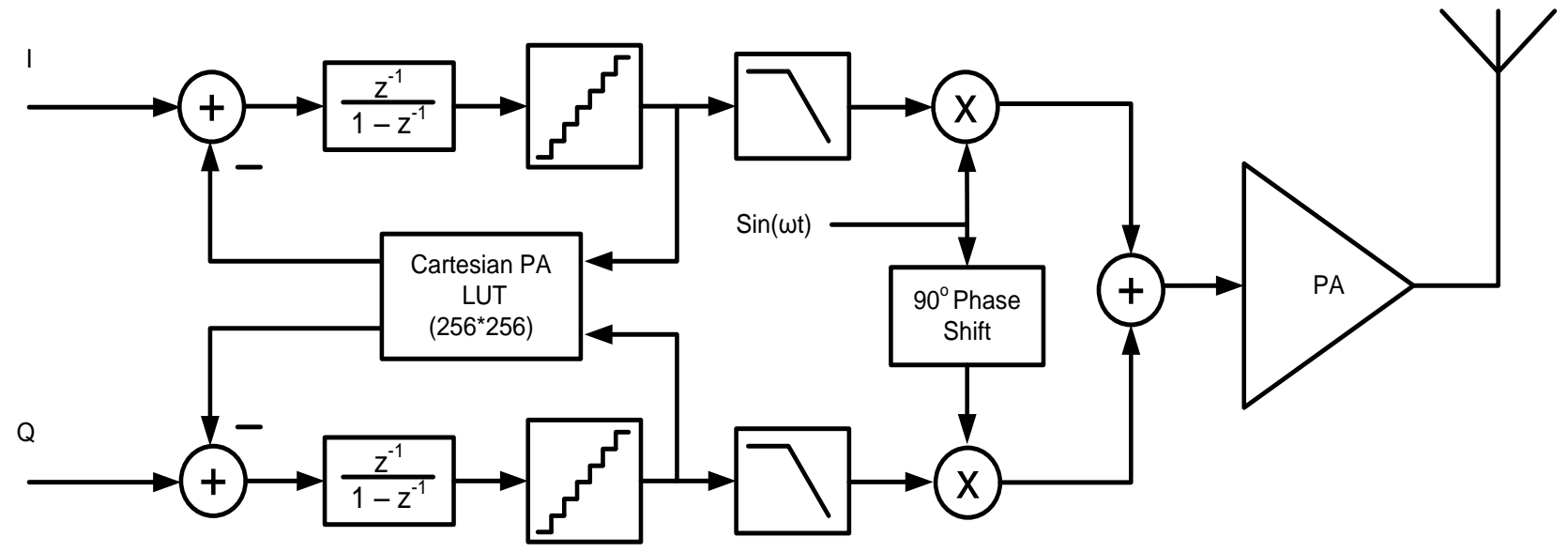

Figure 2 Quadrature implementation with PA LUT

A more in depth but cumbersome model of the power amplifier will negatively affect the critical path of the SDM, reducing the operating frequency of the system which in turn will impact on system performance. The proposed system in this paper uses a Cartesian LUT to implement the PA model, the LUT will operate in a single clock cycle allowing higher operating frequencies to be achieved. The model is trained so that it may operate reliably for multiple standards without the need for re-training. This is a specific advantage for software defined radio where multi standard operation is a common goal to improve spectral efficiency [5].

\section{Digital PRE Distortion WITH Sigma DELTA MODULATORS}

The system in Figure 1 implements a predistortion algorithm for a non-linear PA by placing a pseudo static forward model of the PA in the feedback path of a SDM. The SDM operates by converting the output to a digitised version of the input, however quantisation of the signal increases the noise floor of the output. The SDM is designed to reduce the in band noise, allowing course levels of quantisation to achieve dynamic range similar to finer levels of quantisation within the notch provided by the loop filter. At any instance of time the error of the output relative to the input may be high, however the SDM operates by agitating the error over time using the error to influence future output's .This is achieved by a feedback path from output to input. The error is calculated using the difference between current input and previous output, the loop filter is then updated using this value. The filter shapes the quantisation noise of the output rejecting it from the desired frequency band and spreading it across the remaining spectrum. This causes an increase in the out of band noise floor. The quantisation noise generated from the output is at a constant power level, therefore by increasing the available spectrum the power level of the quantisation noise can be reduced across the entire frequency band. The frequency of operation will however be limited by implementation and circuit constraints.

A standard SDM aims with each iteration to reduce the error between input and output to zero. In Figure 1 once the PA model is placed in the feedback path it modifies the SDM feedback signal by applying the non linear effects of the amplifier. Using the property that the SDM will try to reduce the input - output error to zero the modified modulator structure will compensate for the non linear error of the PA. The output of the SDM is now a pre-distorted version of the input which includes some out of band quantisation noise. The PA model will be implemented using a LUT approach, it will have a finite number of entries leading to additional quantisation noise. Due to the operation of the loop filter the extra quantisation noise does not affect the in band signal, it will be coupled with the out of band quantisation noise which will be filtered out before the amplification stage.

The system aims to reduce the computational complexity incurred through calculating a predistorter weights for the power amplifier. Using a pre-calculated model of the amplifier and placing this in the feedback path of a SDM a pre distorted input to the amplifier is produced. The structure in Figure 2 is a complex SDM structure, the I and Q channels are processed separately as real signals, the feedback path used a common PA model that requires an input from both modulators. The predistortion is carried out at baseband using a low pass sigma delta modulator structure. The low pass filter following the modulator is used to demodulate the output, converting the pulse density modulated (PDM) signal to an analogue signal for frequency up conversion and transmission. Filtering also removes any out of band quantisation noise introduced by the sigma delta modulation process. Quadrature mixing is preformed on the complex signal components to combine them and up convert to a carrier frequency before amplification. 


\section{a) Sigma delta modulator}

Sigma delta modulation performance is defined by three parameters, frequency of operation, modulator order, and the number of quantization levels of the output [6]. In direct analogue to digital conversion the noise floor of the signal is defined by the number of levels of quantization, a sigma delta modulator changes this, using an internal loop filter to push the unwanted quantization noise out of band, increasing the dynamic range of the signal.

In a SDM the number of poles in the system define the order of the modulator and the arrangement of the poles define the type of modulator that is being implemented, either low pass, band pass or high pass. The nonlinearity of the power amplifier can also be described as a system with poles and zeroes. The combined PA-SDM system can no longer be described by the order of the loop filter and the addition of the PA model will change the system response. More to the point, the addition of a non linear PA model in the feedback path of the SDM there is a strong possibility this will cause instability. To ensure the system is robust the lowest possible order loop filter should be implemented. The SDM chosen is a low pass first order modulator, it has a single pole in the loop filter that is on the unit circle, it is the least sensitive SDM structure to instability. Assuming that the amplifier is a stable system, the model should consist of poles and zeroes inside the unit circle, the addition of the 1 st order SDM and PA model should reduce the risk of instability in the system.

To reduce noise in the system the order of quantization must be relatively high. The modulator is chosen to have a 10 bit output quantizer which without the aid of noise rejection from the SDM will achieve $60 \mathrm{~dB}$ of dynamic range. Using a LUT model of the PA reduces the number of computations per iteration to a single memory read operation, allowing for a shortened critical path and therefore the modulator can operate at higher frequencies. Similar to a standard SDM the operating frequency affects the reduction of error introduced by the PA model and as a result the quality of the predistortion signal. To achieve the desired predistortion performance a minimum modulator operating frequency of $400 \mathrm{MHz}$ is required.

\section{b) Power Amplifier Model}

The method chosen to implement the forward PA model is a complex input - output LUT. The model is very compact and is populated using a simple training sequence, the sequence steps through all possible complex inputs in an 8 bit LUT, the training sequence is independent of modulation schemes making it robust to changes in the input modulation schemes. The LUT stores the information in a $2 \mathrm{D} 8$ bit by 8 bit matrix the outputs from the matrix are the corresponding distorted real and imaginary components.

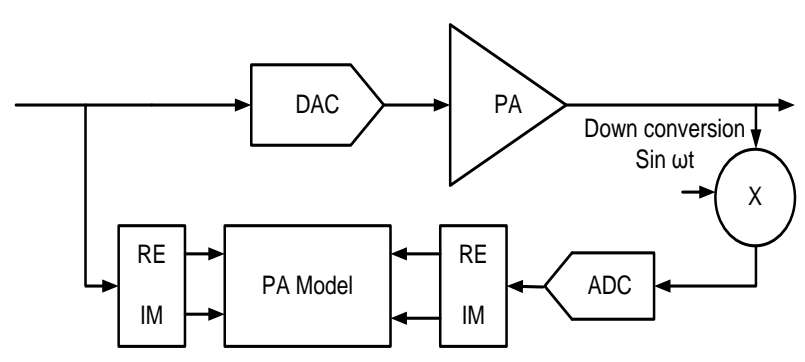

Figure 3 PA model training algorithm

The PA model can be trained both in real time and off line mode. The training sequence does not require calculations to be carried out, the update algorithm uses a quantised version of the input to the amplifier as the address of the LUT and the down converted output of the amplifier will be the data assigned to that address. Filling the LUT requires $256 * 256$ iterations stepping through each of the possible LUT inputs.

\section{SIMULATION}

Simulations were carried out in Matlab with a quadrature sigma delta modulator in low pass configuration as shown in Figure 2. To simulate a pseudo static non linear power amplifier the Saleh model was chosen [7]. The Saleh model is a set of two parameter formulas that develops a polar model of a non linear amplifier. Equations (1) and (2) are used to calculate the phase and amplitude distortion applied by the Saleh model. The parameters $\left(\alpha_{a}, \beta_{a}\right)$ define the amplitude curve $A(r)$ of the model and parameters $\left(\alpha_{\phi}, \beta_{\phi}\right)$ define phase curve $\phi(r)$, the variable $r$ is the absolute amplitude of the input signal. The model is an AMAM, AMPM equivalent of an amplifier, applying the training sequence to the amplifier extracts its Cartesian equivalent.

$$
\begin{aligned}
& A(r)=\alpha_{a} r /\left(1+\beta_{a} r^{2}\right) \\
& \phi(r)=\alpha_{\phi} r^{2} /\left(1+\beta_{\phi} r^{2}\right)
\end{aligned}
$$

The system was tested using three signals with different modulation schemes and bandwidths. A QPSK and a 16 QAM signal both with a bandwidth of $5 \mathrm{MHz}$ and a WCDMA signal with a $3.84 \mathrm{MHz}$ bandwidth. The QPSK and QAM signals were used as a comparison between signal standards with similar bandwidths, the WCDMA signal tests the effect of differing bandwidth on the system. The modulator will be operated at a constant frequency of $400 \mathrm{MHz}$. This increase in bandwidth will reduce the oversampling ratio, from SDM theory this will lead 
to reduced dynamic range from the modulator output.

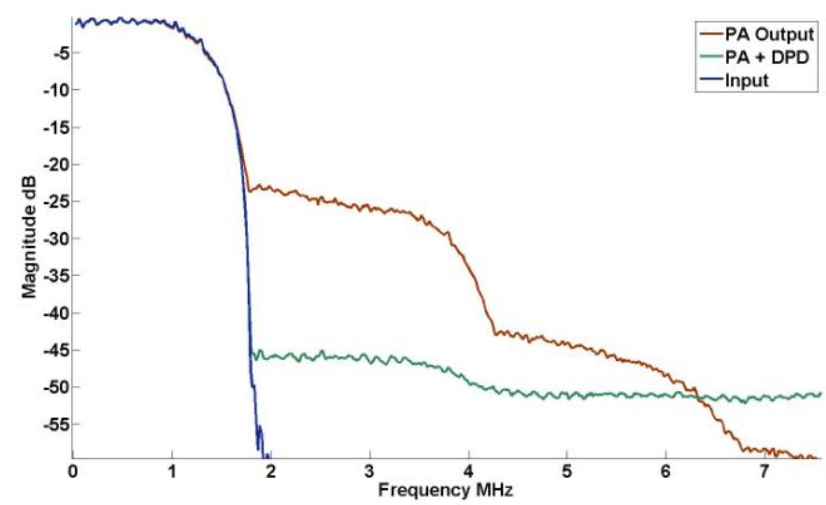

Figure 4 QAM 16 input signal at baseband compared to PA output with and without DPD

It can be seen from Figures 4-6 that the modulation schemes affect the extent and shape of the distortion applied to the PA output. QAM and WCDMA signals vary in amplitude and phase, it is clear that they have similar distortion characteristics despite the differences in bandwidth. The QPSK signal in Figure 6 has constant amplitude compared to the QAM and WCDMA signal this is a fundamentally different modulation scheme. In this paper the amplitude of the QPSK signal was chosen to be the maximum input amplitude of the amplifier. The distortion of the signal is much stronger when compared to the QAM of WCDMA signal, however the system in this paper is able to pre distort the signal to improve the output without the need to re train the LUT.

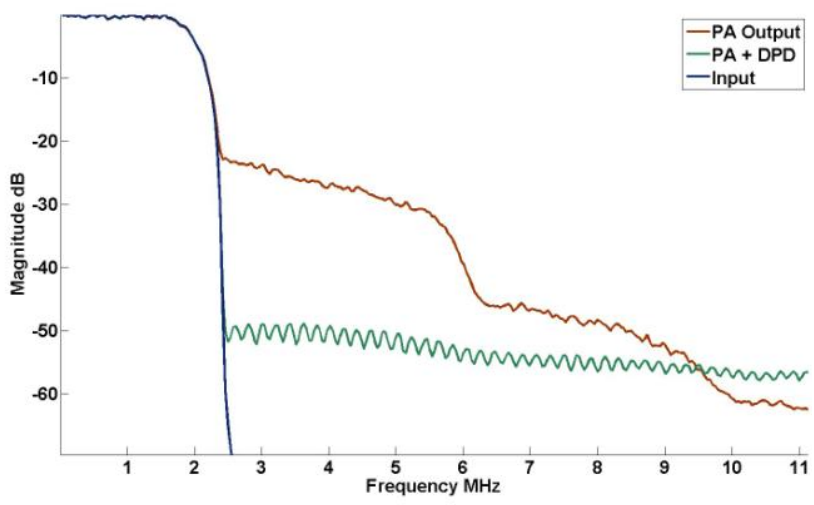

Figure 5 WCDMA input signal at baseband compared to PA output with and without DPD.

\section{RESUlTS}

Simulation results show that the pre distortion method reduces the inter modulation effects introduced by the amplifier. In Figure 5 the WCDMA signal with a bandwidth of $3.84 \mathrm{MHz}$ is used to test the system, the PA causes strong 3rd order inter modulation products to occur in the output signal. The predistortion method is able to reduce the adjacent channel power by $28 \mathrm{~dB}$ and the resulting performance is sufficient to meet spectral mask performance requirements for 3GPP base station transmission. For QAM and QPSK signals there is a reduction of $19 \mathrm{~dB}$ and $18 \mathrm{~dB}$ respectively. The reduced performance of the system with wider bandwidth signals follows the theory of SDM performance.

Figure 4 shows a slight increase in the noise floors of the signals out of band. This is due to the introduction of quantisation noise from the SDM output and the PA model. This noise however is uniform and below the level of the amplifier distortion. The modulator noise further out of band is reduced by the demodulation process, output filters would be required to remove the noise close to the signal band.

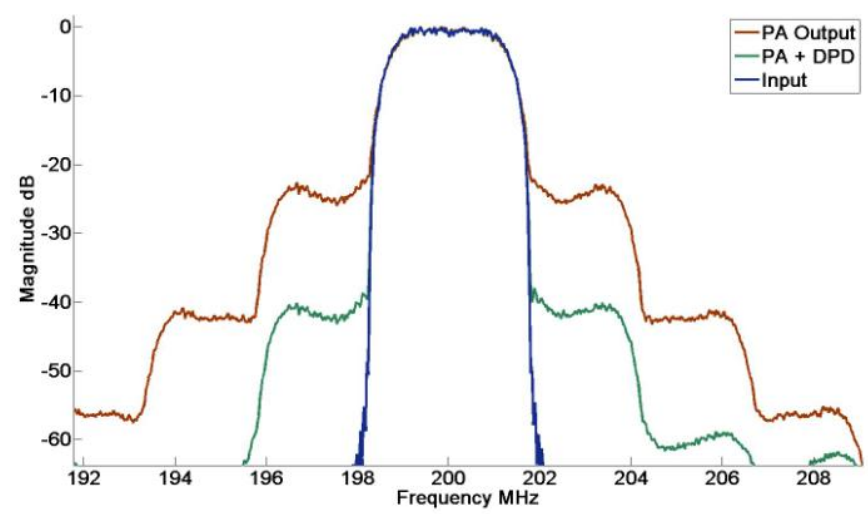

Figure 6 QPSK input signal at carrier frequency compared to PA output with and without DPD.

\section{CONCLUSION}

This method of predistortion offers advantages of reduced computational complexity in the system as well as a robust structure for pre distortion of RF PA's. The results in this paper show an improvement of up to $28 \mathrm{~dB}$ in the case of a WCDMA signal. This improvement in system performance is enough for the signal to meet $3 \mathrm{GPP}$ spectral mask requirements. The reduction in distortion is adequate in all 3 signal standards while the PA model was kept constant throughout the simulations. The system has advantages in multi standard software defined radio where constant re-training would negatively impact on the system.

\section{ACKNOWLEDGEMENTS}

This project is funded by the HEA under PRTLI5 and is being co-funded by the Irish Government and the EU under Ireland's Structural Funds Programmes 
2007-2013. The authors would also like to gratefully acknowledge this support of Science Foundation Ireland under grant number 08/CE/I1523.

\section{REFERENCES}

[1] C. SungWon, J. W. Holloway, and J. L. Dawson, "Energy-Efficient Digital Predistortion With Lookup Table Training Using Analog Cartesian Feedback," Microwave Theory and Techniques, IEEE Transactions on, vol. 56, pp. 2248-2258, 2008.

[2] H. H. Boo, C. Sung Won, and J. L. Dawson, "Adaptive Predistortion Using a Sigma Delta Modulator for Automatic Inversion of Power Amplifier Nonlinearity," Circuits and Systems II: Express Briefs, IEEE Transactions on, vol. 56, pp. 901-905, 2009.

[3] S. Chung and J. L. Dawson, "Digital predistortion using quadrature delta-sigma modulation with fast adaptation for WLAN power amplifiers," in Microwave Symposium Digest (MTT), 2011 IEEE MTT-S International, pp. 1-1.

[4] Tripath, "Method and apparatus for oversampled, noise-shaping, mixed-signal processing," 1996.

[5] O. Zlydareva and C. Sacchi, "A software radio architecture for the baseband level of the multistandard user terminal: Design methodology and computational assessment," in Wireless Days (WD), 2010 IFIP, pp. 1-5.

[6] R. Schreier and G. C. Temes, Understanding Delat-Sigma Data Converters, 2005.

[7] M. O'Droma, S. Meza, and Y. Lei, "New modified saleh models for memoryless nonlinear power amplifier behavioural modelling," Communications Letters, IEEE, vol. 13, pp. 399401, 2009. 